\title{
Anwendung der Induzierten Polarisation in der Graphitexploration
}

\author{
Adrián Flores-Orozco', Timea Katona', Lukas Aigner'1, Matthias Steiner' ${ }^{1}$ und Alexander Römer ${ }^{2}$ \\ 'Department für Geodäsie und Geoinformation, Forschungsbereich Geophysik, TU Wien, Wien, Österreich \\ ${ }^{2}$ Fachabteilung Geophysik, Geologische Bundesanstalt (GBA), Wien, Österreich
}

Eingegangen 15. Februar 2021; angenommen 1. März 2021; online publiziert 22. März 2021

\begin{abstract}
Zusammenfassung: Diese Studie präsentiert die Anwendung der Induzierten Polarisation (IP) für die Charakterisierung einer Graphitlagerstätte. Basierend auf Labormessungen zeigen wir die Sensitivität von IP-Messungen hinsichtlich Veränderungen der Korngröße und der spezifischen Oberfläche von Graphit in Gesteinsproben und auf der Feldskala. Des Weiteren demonstrieren wir, dass IPFeldmessungen eine klarere Abgrenzung von graphitreichen Untergrundbereichen ermöglichen als die alleinige Bestimmung der elektrischen Leitfähigkeit.
\end{abstract}

Schlüsselwörter: Graphitexploration, Induzierte Polarisation, Komplexe elektrische Leitfähigkeit, Geophysik, Mineralische Rohstoffe

The Use of the Induced Polarization Method for the Characterization of Graphite Ores

Abstract: We demonstrate here the application of the induced polarization (IP) method for the characterization of graphite. Based on laboratory measurements, we demonstrate that IP measurements are sensitive to changes in the grain size and surface area of graphite in rocks samples and for measurements at the field scale. Built on such results, we demonstrate that IP field imaging results permit a clearer delineation of graphite-rich areas than the use of only electrical conductivity results.

Keywords: Graphite exploration, Induced polarization, Complex conductivity, Geophysics, Raw materials

\footnotetext{
Ass. Prof. Dr.-habil. A. Flores-Orozco ( $\square)$

Department für Geodäsie und Geoinformation,

Forschungsbereich Geophysik,

TU Wien,

Wiedner Hauptstraße 8-10,

1040 Wien, Österreich

adrian.flores-orozco@geo.tuwien.ac.at
}

\section{Einleitung}

Die Induzierte Polarisation (IP) ist eine geophysikalische Methode, die aufgrund ihrer Sensitivität für die geometrischen und geochemischen Eigenschaften von Gesteinen und Sedimenten in der Prospektion von Rohstoffen eingesetzt wird. IP-Messungen basieren auf einer 4-ElektrodenAnordnung, wobei zwei Elektroden für das Einspeisen von Strom in den Untergrund verwendet werden, während mit dem zweiten Elektrodenpaar das resultierende elektrische Potenzial gemessen wird [1, 2]. Bestimmte Mineralien (FeS und Graphit) gelten als elektrische Leiter, da sie die freie Bewegung von Elektronen während der Stromeinspeisung erlauben und somit einen niedrigen spezifischen Widerstand $(\rho)$ bzw. eine hohe elektrische Leitfähigkeit $(\sigma=1 / \rho)$ aufweisen. Zudem verursacht ein externes elektrisches Feld die Bewegung von positiven und negativen Ladungen innerhalb des elektrischen Leiters in unterschiedliche Richtungen, wodurch es zunächst zur Polarisierung des elektrischen Leiters und in weiterer Folge zur Polarisierung des Elektrolyts (das Porenwasser) kommt. Verschwindet das externe elektrische Feld, kehren die elektrischen Ladungen in ihren Gleichgewichtszustand zurück, und es kommt zur Ausbildung eines sekundären elektrischen Feldes, welches den messbaren IP-Effekt verursacht. Dieser Polarisationseffekt ist umso größer je höher der Anteil an elektrischen Leitern (z. B. Eisen oder Graphit) im Untergrund ist [1-3]. Dabei ist jedoch zu beachten, dass größere Minerale einen langsameren Polarisationsprozess verursachen als kleinere Minerale, da sich die Ladungen über unterschiedliche Distanzen bewegen müssen. Um dies zu berücksichtigen, können IP-Messungen mit Wechselstrom bei niedrigen Frequenzen $(<1 \mathrm{kHz})$ durchgeführt werden (FDIP). Durch Messungen mit verschiedenen Frequenzen können Informationen hinsichtlich der Frequenzabhängigkeit des spezifischen elektrischen Widerstandes und der Polarisation gewonnen werden (Spektrale IP, oder SIP), welche von der Textur der Minerale abhängig ist. SIP-Messungen können auch mit Gleichstrom durchgeführt werden (direct current IP - DCIP), entweder durch die Verwendung unterschiedlicher Pulslän- 
gen oder durch die Aufzeichnung der gesamten Strom- und Spannungssignale mit hoher Auflösung. Moderne Inversionsalgorithmen ermöglichen die Anwendung von tomographischen Messanordnungen bestehend aus hunderten Elektroden, um die vertikalen und lateralen Änderungen in den elektrischen Eigenschaften des Untergrundes zu bestimmen [1, 2]. Die Inversionsergebnisse werden üblicherweise in Form der komplexen elektrischen Leitfähigkeit dargestellt:

$$
\sigma^{*}=\sigma^{\prime}+i \sigma^{\prime \prime}=|\sigma| \cdot e^{i \varphi}
$$

wobei $i=\sqrt{-1}$ die imaginäre Einheit ist. Der Realteil $\sigma^{\prime}$ dieser komplexen Größe beschreibt in erster Linie elektrische (ohmsche) Verluste im Medium und stimmt weitestgehend mit der elektrischen Leitfähigkeit überein. Der Imaginärteil $\sigma^{\prime \prime}$ beschreibt elektrische Aufladungs- oder Polarisationsprozesse im Untergrund, d. h. dessen kapazitive Eigenschaften. In den meisten praktischen Anwendungen sind die Phasendifferenzen vernachlässigbar klein $(<100 \mathrm{mrad})$, sodass die Annahmen $\sigma^{\prime} \approx|\sigma|$ und $\varphi \approx \sigma^{\prime \prime} / \sigma^{\prime}$ zulässig sind. Im weiteren Verlauf dieser Studie beschreiben wir die elektrische Leitfähigkeit und die Polarisation durch die $|\sigma|$ und $\varphi$ Parameter.

Im Fall von metallfreien Sedimenten wird die elektrische Leitfähigkeit hauptsächlich durch Porosität, Sättigung und Elektrolytleitfähigkeit sowie durch die Porenkonnektivität bestimmt. Im Fall von eingelagerten elektrischen Leitern (z. B. Graphit) wird die elektrische Leitfähigkeit von der elektronischen Matrixleitfähigkeit dominiert, unabhängig von Sättigung und Porosität der Sedimente. Aufgrund der Polarisation von freien Ladungsträgern in elektrischen Leitern sowie innerhalb der elektrischen Doppelschicht zwischen Porenfluid und Porenmineral sind diese Leiter durch einen hohen Polarisationseffekt charakterisiert. Darüber hinaus wurde in verschiedenen Studien beobachtet, dass die Frequenzabhängigkeit der Polarisation von Größe, Volumen und Textur der elektrisch leitfähigen Minerale abhängt [3].
Aufgrund der Vielzahl an möglichen Fehlerquellen [2], welche die SIP-Messungen beeinflussen können, beschränken sich bisherige Anwendungen hauptsächlich auf Laboruntersuchungen. Zudem entstehen bei Messungen in elektrisch leitfähigen Materialen geringe elektrische Spannungen, wodurch es zu einer weiteren Verschlechterung des Signal-Rausch-Verhältnisses (S/N) kommt. Darüber hinaus ist die Interpretation der SIP-Ergebnisse nicht immer eindeutig möglich. In dieser Studie sollen die Vorteile der SIP hinsichtlich der Charakterisierung von Graphitlagerstätten demonstriert werden. Basierend auf dem Vergleich von SIP. Signaturen auf der Feld- und der Laborskala diskutieren wir Parameter, welche hinsichtlich der Frequenzabhängigkeit der elektrischen Eigenschaften relevant sind.

Die in dieser Studie präsentierten Ergebnisse basieren auf Untersuchungen einer ehemaligen Graphitlagerstätte in der Nähe von Zettlitz (Österreich). Die Daten entstammen einer Forschungskooperation zwischen der TU Wien und der Geologischen Bundesanstalt (GBA), die durch das Ministerium für Wissenschaft, Forschung und Wirtschaft im Rahmen der Rohstoff-Initiative gefördert wurde.

\section{Labormessungen: Proof of Concept}

Um die Frequenzabhängigkeit der elektrischen Eigenschaften zu untersuchen, wurden Labormessungen an verschiedenen Proben mit dem Messsystem PSIP (Ontash \& Ermac) der TU Wien durchgeführt. Dies ermöglicht es, die Sensibilität der SIP auf Änderungen der Korngröße und der spezifischen Oberfläche der elektrischen Leiter zu demonstrieren. Labormessungen wurden sowohl an Lockermaterialproben als auch an Festgesteinsproben durchgeführt. Für erstere wurden Gesteinsproben aus dem Untersuchungsgebiet im Labor zerkleinert, um Körner mit unterschiedlicher Größe zu erhalten: fein $(<0,45 \mathrm{~mm})$, mittel $(0,45-1 \mathrm{~mm})$, grob $(1-10 \mathrm{~mm})$ und groß $(>10 \mathrm{~mm})$. Diese zerkleinerten Körner wurden in Form von Säulenexperimenten
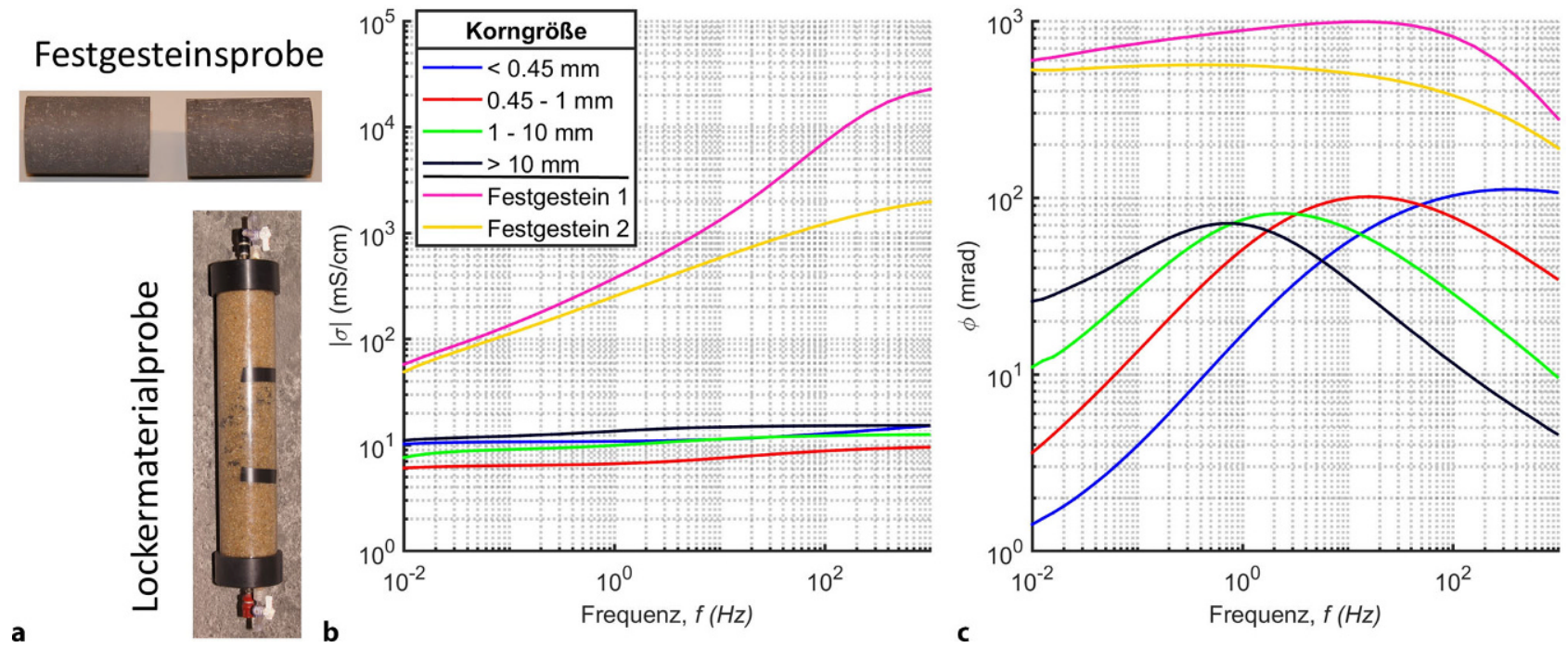

Abb. 1: Ergebnisse der im Labor gemessenen Spektralen Induzierten Polarisation (SIP). SIP-Labormessungen wurden für Festgesteins- und Lockermaterialproben (a) durchgeführt und zeigen die Frequenzabhängigkeit der elektrischen Leitfähigkeit (b) und der Polarisation (c) 
untersucht, wobei die Dimensionen der Messsäule durch einen Plexiglaszylinder mit $30 \mathrm{~cm}$ Länge und $2,5 \mathrm{~cm}$ Radius vorgegeben sind. Zwei Plattenelektroden mit $5 \mathrm{~cm}$ Durchmesser ermöglichten eine homogene Verteilung der Stromdichte, und die Spannung wurde mit zwei nicht-polarisierbaren Elektroden ( $10 \mathrm{~cm}$ Abstand) in der Mitte der Probe gemessen, wobei SIP Messungen bei 64 verschiedenen Frequenzen zwischen 0,01 und $20.000 \mathrm{~Hz}$ aufgezeichnet wurden. Für die Festgesteinsproben wurden Bohrkerne aus den Gesteinsproben entnommen und mit einer äquivalenten Messanordnung untersucht, wobei die Zylinderdimensionen angepasst wurden ( $6 \mathrm{~cm}$ Länge, Durchmesser $3,8 \mathrm{~cm})$.

Die IP-Spektren der komplexen elektrischen Leitfähigkeit von Lockermaterial- und Festgesteinsproben sind in Abb. 1 dargestellt und ermöglichen drei zentrale Beobachtungen:

1. Die Leitfähigkeit $(|\sigma|)$ der Lockermaterialproben zeigt eine geringe Frequenzabhängigkeit im Bereich von $10 \mathrm{mS} / \mathrm{cm}$. Dies ist aufgrund der verwendeten niedrigen Frequenzen zu erwarten.

2. Die Polarisation $(\varphi)$ der Lockermaterialproben zeigt eine deutliche Frequenzabhängigkeit mit einem klaren Frequenzmaximum für jede Korngröße. Diese Frequenzmaxima liegen im Bereich von $1-300 \mathrm{~Hz}$ und verschieben sich mit abnehmender Korngröße zu höheren Frequenzen, was dem von theoretischen Modellen [3] vorhergesagten Verhalten entspricht.

3. Die Festgesteinsproben zeigen aufgrund des höheren Graphitgehalts eine um eine Magnitude erhöhte Leitfähigkeit und Polarisation $(\varphi)$. Das Frequenzmaximum der Polarisation $(\varphi)$ liegt bei niedrigen Frequenzen im Bereich von 0,1 und $30 \mathrm{~Hz}$ was auf Graphitminerale mit Korngrößen zwischen 1 und $10 \mathrm{~mm}$ hinweist.

Abb. 1 demonstriert den Vorteil von SIP-Messungen gegenüber der alleinigen Bestimmung der elektrischen Leitfähigkeit: (1) Mehr Daten für eine verbesserte Interpretation. (2) Die Frequenzabhängigkeit ermöglicht die Unterscheidung zwischen verschiedenen Korngrößen und somit verschiedenen spezifischen Oberflächen (d. h. Kornoberflächen).

\section{Feldmessungen}

Obwohl Labormessungen wertvolle Informationen liefern, sind diese nur begrenzt auf die Feldskala übertragbar. Daher wurden im Bereich der Graphitlagerstätte Zettlitz Messungen entlang zweier Ost-West-orientierter Profile durchgeführt: (a) Profil Z1 besteht aus 96 Elektroden mit $10 \mathrm{~m}$ Abstand; (b) Profil Z2 befindet sich in der Mitte von Profil Z1 und besteht aus 64 Elektroden mit $5 \mathrm{~m}$ Abstand. Die Verwendung zweier verschiedener Messanordnungen soll demonstrieren, wie durch Variieren des Elektrodenabstandes die Auflösung und die Untersuchungstiefe verbessert werden können. Die Daten wurden mit dem Messsystem DAS-1 (Multi-Phase Technologies, LLC) der TU Wien gemessen.

Abb. 2 zeigt IP-Ergebnisse für die Profile Z1 und Z2. Messungen entlang von Z1 wurden sowohl mit Direktstrom als auch mit Wechselstrom (DCIP und FDIP) durchgeführt, wodurch gezeigt werden kann, dass die Ergebnisse beider Methoden konsistent sind. Um DCIP und FDIP vergleichbar zu machen, wurde die Aufladbarkeit linear zu scheinbaren Phasen transformiert, um beide Datensätze mit demselben Algorithmus invertieren zu können [1]. Die resultierenden Modelle der Leitfähigkeit (Abb. 2a, c) zeigen, dass beide Methoden, abgesehen von geringen Variationen bis Profilmeter 200, ein konsistentes Bild des Untergrunds liefern. Im Fall des Polarisationseffekts (Abb. 2b, d) sind die Ergebnisse der beiden Methoden ebenfalls größtenteils konsistent, jedoch wurden für zwei Bereiche unterschiedliche Charakteristika gefunden. Das DCIP-Polarisationsmodel zeigt am Beginn des Profils (100-200 m) eine Anomalie, die im FDIPPolarisationsmodell nicht aufgelöst wurde. Des Weiteren zeigt Abb. 2b (FDIP-Polarisationsmodell) eine tiefliegende polarisierbare Anomalie $(>20 \mathrm{~m})$ im Bereich von 200 bis $500 \mathrm{~m}$ in Profilrichtung.

Die im DCIP-Polarisationsmodel sichtbare Anomalie am Beginn des Profils steht in keinem Zusammenhang mit den Veränderungen in der Leitfähigkeit (vgl. dazu Abb. 2a). Da DCIP-Daten nach der Stromeinspeisung gemessen werden und daher empfindlicher auf externe Störquellen reagieren, kann diese Anomalie als Artefakt aufgrund eines geringen $\mathrm{S} / \mathrm{N}$ betrachtet werden. Die tiefliegende Anomalie im FDIP-Polarisationsmodell deutet auf eine polarisierbare Struktur hin, wobei deren Geometrie aufgrund der großen Tiefe nicht gänzlich korrekt aufgelöst werden kann. Der Grund dafür ist das oberflächennah abgelagerte Graphiterz, dessen hohe Leitfähigkeit die Stromdichte in größeren Tiefen stark limitiert. Dementsprechend ist der Einfluss von zufälligen Messfehlern auf Messungen mit großem Elektrodenabstand bzw. großem Abstand zwischen Stromund Spannungsdipolen besonders ausgeprägt, wodurch die tatsächlich erreichbare Untersuchungstiefe reduziert wird (die gebräuchliche Daumenregel, wonach die Untersuchungstiefe ca. einem Drittel der Profillänge entspricht, trifft hier nicht zu).

Abb. 2e-h zeigen die SIP-Ergebnisse für Profil Z2 für zwei unterschiedlichen Frequenzen $(0,5$ und $15 \mathrm{~Hz})$. Die entsprechenden elektrischen Modelle (Leitfähigkeit und Polarisation) des Untergrunds sind konsistent mit den Ergebnissen für das Profil Z1 und zeigen zwei weitere polarisierbare Anomalien:

1. $350-450 \mathrm{~m}$ in Profilrichtung; ca. $35 \mathrm{~m}$ dick; max. $~ 50 \mathrm{mrad}$ bei einer kritischen Frequenz von $0,5 \mathrm{~Hz}$

2. $500-550 \mathrm{~m}$ in Profilrichtung; ca. $20 \mathrm{~m}$ dick; $\mathrm{max} \sim 80 \mathrm{mrad}$ bei einer kritischen Frequenz von $0,5 \mathrm{~Hz}$

3. Die höchauflösenden SIP-Ergebnisse (Abb. 2f, h) zeigen, dass der Polarisationseffekt das Maximum bei einer niedrigen Frequenz erreicht $(0,5 \mathrm{~Hz})$. Dies deutet daraufhin, dass im Untergrund ein hoher Anteil an Graphit mit Korngrößen $>5 \mathrm{~mm}$ vorhanden ist. Die Leitfähigkeitswerte sind konsistent für Profil Z1 (Abb. 2a, c) und Profil Z2 (Abb. 2e, g).

Beide Anomalien weisen auf graphitreiche Bereiche hin, welche durch die alleinige Betrachtung der Leitfähigkeit nicht aufgelöst werden können. Außerdem deuten die Variationen der Polarisierbarkeit innerhalb der Anomalien 

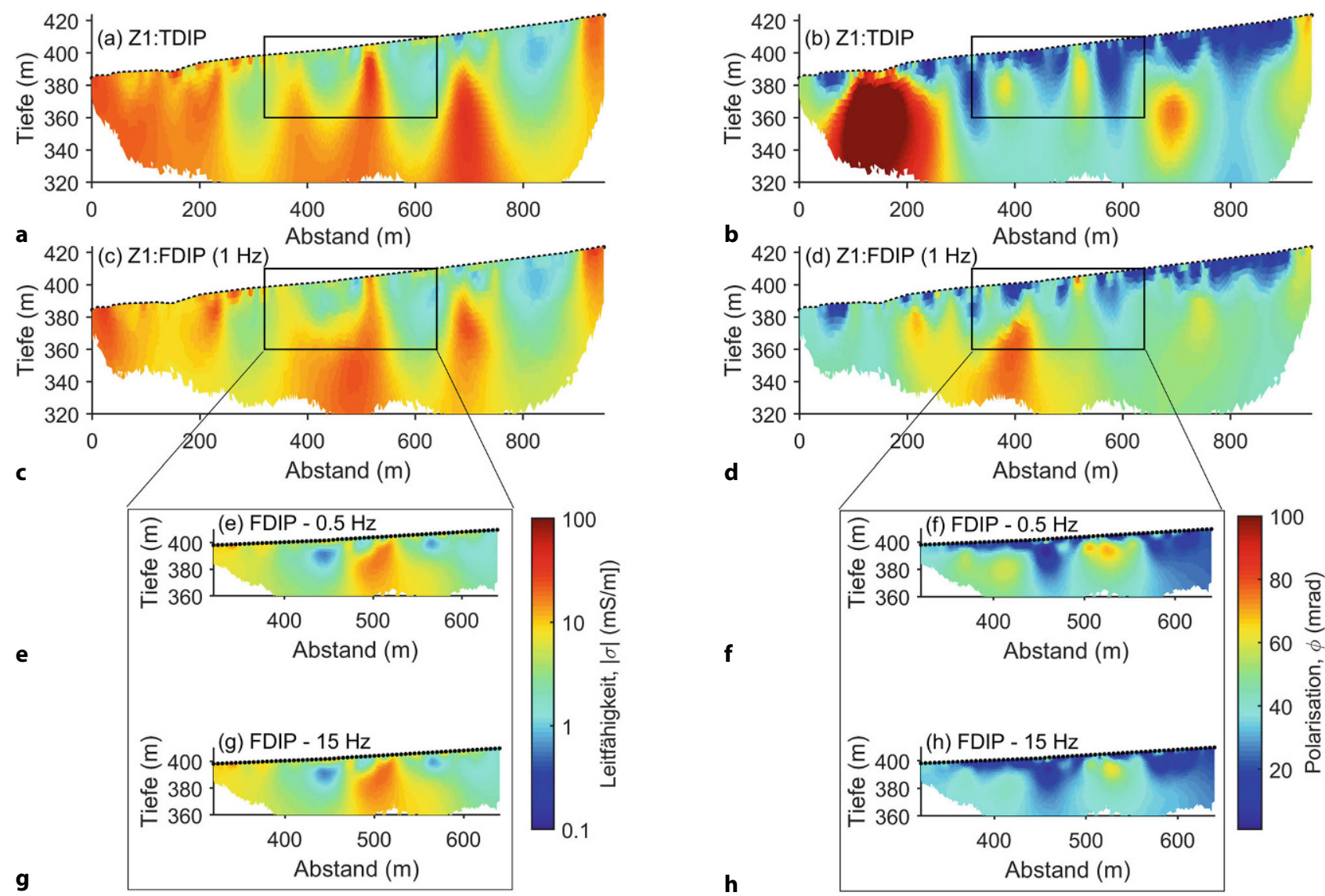

Abb. 2: Tomographische Ergebnisse der Feldmessungen, dargestellt als elektrische Leifähigkeit und Polarisation für die DCIP entlang von Profil Z1 (a und b), die FDIP entlang von Profil Z2 (c und d) und die SIPentlang von Profil Z2 (e-h)

darauf hin, dass die erste (größere) Anomalie einen kleineren Anteil an Graphiterz enthält als die zweite (kleinere) Anomalie. Für eine quantitative Analyse der Ergebnisse wäre eine Analyse des Materials aus tiefen Bohrlöchern im Bereich dieser Anomalien notwendig, was jedoch den Rahmen dieser Untersuchung übersteigt.

\section{Fazit und Ausblick}

In dieser Studie konnten wir zeigen, dass die Interpretation von geoelektrischen Ergebnissen durch die Berücksichtigung von IP-Messungen verbessert werden kann. Die IP liefert räumlich kontinuierliche Informationen basierend auf nicht-invasiven Messungen an der Erdoberfläche, wodurch sie eine geeignete Methode für die hochauflösende Charakterisierung von Graphitlagerstätten darstellt, die mit den herkömmlichen Methoden nicht erreicht werden kann. Dennoch werden direkte Informationen (z. B. aus Boden-/Wasserproben) für die Kalibrierung der petrophysikalischen Modelle benötigt, welche die elektrischen Eigenschaften mit den Zielparametern in Beziehung setzen. Laboruntersuchungen helfen, die im Feld beobachteten IPSignaturen (detaillierter) zu analysieren und die Frequenzabhängigkeit mit der Korngröße in Beziehung zu setzen.
Danksagung. Diese Studie wurde durch die beiden Projekte POTHAL und EXPLOGRAF dankenswerter Weise vom Bundesministerium für Bildung, Wissenschaft und Forschung finanziell unterstützt.

Funding. Open access funding provided by TU Wien (TUW).

Open Access Dieser Artikel wird unter der Creative Commons Namensnennung 4.0 International Lizenz veröffentlicht, welche die Nutzung, Vervielfältigung, Bearbeitung, Verbreitung und Wiedergabe in jeglichem Medium und Format erlaubt, sofern Sie den/die ursprünglichen Autor(en) und die Quelle ordnungsgemäß nennen, einen Link zur Creative Commons Lizenz beifügen und angeben, ob Änderungen vorgenommen wurden.

Die in diesem Artikel enthaltenen Bilder und sonstiges Drittmaterial unterliegen ebenfalls der genannten Creative Commons Lizenz, sofern sich aus der Abbildungslegende nichts anderes ergibt. Sofern das betreffende Material nicht unter der genannten Creative Commons Lizenz steht und die betreffende Handlung nicht nach gesetzlichen Vorschriften erlaubt ist, ist für die oben aufgeführten Weiterverwendungen des Materials die Einwilligung des jeweiligen Rechteinhabers einzuholen.

Weitere Details zur Lizenz entnehmen Sie bitte der Lizenzinformation auf http://creativecommons.org/licenses/by/4.0/deed.de.

\section{Literatur}

1. Flores-Orozco, A.; Gallistl, J.; Steiner, M. Brandstätter, C.; Fellner, J.: Mapping biogeochemically active zones in landfills with induced polarization imaging: The Heferlbach landfill, Waste Management, 107 (2020), pp121-132 
2. Flores Orozco, A.; Aigner, L.; Gallistl, J.: Investigating cable effects in spectral induced polarization imaging at the field scale using multicore and coaxial cables, Geophysics, 86 (2020), no. 1, pp1-49

3. Bücker, M.; Flores Orozco, A. F.; Kemna, A.: Electrochemical polarization around metallic particles-Part 1: The role of diffuse-layer and volume-diffusion relaxation, Geophysics, 83 (2018), no. 4, pp E203-E217

Hinweis des Verlags. Der Verlag bleibt in Hinblick auf geografische Zuordnungen und Gebietsbezeichnungen in veröffentlichten Karten und Institutsadressen neutral. 